\title{
The Need for a Reference Self-Diffusion Mobility Database for the Pure Elements
}

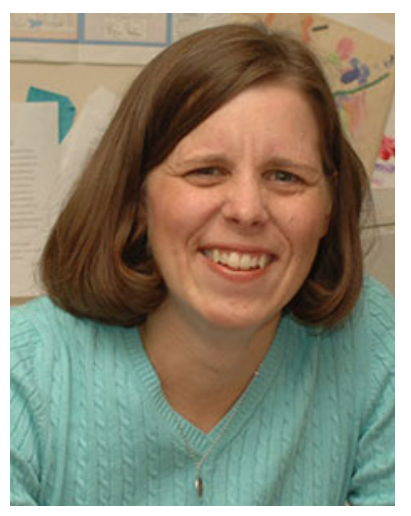

The increased focus on integrated computational materials engineering (ICME), especially through the Materials Genome Initiative (MGI) ${ }^{\dagger}$ has amplified the need to develop CALPHADbased $^{*}$ multicomponent diffusion mobility databases for a variety of applications, ranging from structural $\mathrm{Ti}$ and $\mathrm{Mg}$ alloys to materials for photovoltaic absorber materials. Essential for the development of both commercial and non-commercial multicomponent diffusion mobility databases (e.g. National Institute of Standards and Technology (NIST) Ni mobility database ${ }^{[1]}$ ) are evaluations of the mobilities in the unary, binary and ternary systems, as initially demonstrated by Andersson and Ågren. ${ }^{[2]}$ The growth of published diffusion mobility descriptions and the number of descriptions for self-diffusion of a given pure element has increased significantly in the past 5 years. With this increasing publication rate multiple selfdiffusion mobilities for the same pure element have been published. For example, currently in the published literature there three published diffusion mobility assessments for the selfdiffusion of $\mathrm{Al}$ in the fcc phase ${ }^{[3-5]}$ and two for $\mathrm{V}$ in the bcc phase. ${ }^{[6,7]}$ The existence of multiple published self-diffusion assessments for the same element has led to different binary and ternary assessments choosing different values for the self-diffusion mobility of given pure component. As consequence of this, it becomes difficult to combine various binary and ternary assessments to build needed multicomponent diffusion mobility databases. For example, if one researcher chooses a specific functional description, $A^{*}$, for the self-diffusion of $A$ in the $\alpha$ phase for use in the diffusion mobility description of the $A-B$ system and another researcher describes the self-diffusion of $A$ in the $A$ - $C$ system using a functional description of $A^{\prime}$, then the two binary descriptions cannot be combined to describe the $A-B-C$ system. To combine the two description requires that one of the binary descriptions must be re-evaluated so that self-diffusion of $A$ in both systems equals either $A^{*}$ or $A^{\prime}$.

A similar problem was faced when the first CALPHAD multicomponent thermodynamic databases were being developed and multiple lattice stabilities existed for the free energies of the pure elements. In 1991 to resolve this issue, Dinsdale ${ }^{[8]}$ published the lattice stabilities for the condensed phase for 78 elements. These lattice stabilities are functions of temperature and pressure that describe the variation in the free energy relative to the enthalpy of a defined reference phase and the difference between the free energy and various phases. This work has served as a foundation for the development of multicomponent databases as demonstrated by the over 2000 citations since 1991. A similar type of effort is required for the efficient development of multicomponent diffusion mobility databases. For these mobility databases, a self-consistent database that includes temperature and pressure functional descriptions of the mobility of the pure elements is needed.

Efforts to build such a reference database have been initiated. In January 2009, a group of participants from the NIST Diffusion Workshop series began work to establish an initial set of recommendations for the self-diffusion mobilities for fcc-Al, fcc-Cu, bcc-Cr, fcc-Ni and fcc and bcc Fe. The group reviewed the current experimental data and published self-diffusion mobility assessments and at The Minerals, Metals and Materials Society (TMS) 2011 annual meeting agreed a set of reference values for the six self-diffusion mobilities. (http://www.nist.gov/mml/msed/thermodynamics_kinetics/Diffusion-Workshop-Group.cfm Note this website will be updated when new reference values are accepted.)

While the development of complete set of reference self-diffusion mobilities for the pure elements is not yet complete, I urge those in the community working to develop additional diffusion mobility assessments, of which many are needed, to consider the following before publishing a new pure element self-diffusion mobility assessment:

- Use an available established recommended reference self-diffusion mobility description when possible.

- If there are no established reference descriptions, review the literature to ensure that a previous assessment has not been already been published, before publishing a new description.

- If a previous description has been published, then it clearly demonstrate why a new assessment is needed (i.e. new experimental data has been published since the last description was published).

The development of reference database is a time consuming process of which most of the time is spent searching for and re-analyzing data already published. This development of this reference data will also include the development of a publically

${ }^{\dagger}$ http://www.whitehouse.gov/mgi.

*For a given phase based property, CALPAD-based multicomponent databases are developed by combining unary, binary and ternary descriptions of the composition, temperature and pressure dependences together to predict the behavior in a multicomponent system. 
available data repository with all of the evaluated experimental data. This will hopefully enable more efficient updates to the reference values in the future. I look forward to when this community resource is available and can be used for the efficient development of multicomponent CALPHAD-based diffusion mobility databases.

\section{References}

1. C.E. Campbell, W.J. Boetttinger, and U.R. Kattner, Development of a Diffusion Mobility Database for Ni-Based Superalloys, Acta Mater., 2002, 50, p 775-792

2. J.-O. Andersson and J. Ågren, Models for Numerical Treatment of Multicomponent Diffusion in Simple Phases, J. Appl. Phys., 1992, 72, p $1350-1355$

3. A. Engström and J. Ågren, Assessment of Diffusional Mobilities in Face-centered Cubic Ni-Cr-Al Alloys, Z. Metallkunde, 1996, 87, p $92-97$

4. Y.W. Cui, K. Oikawa, R. Kainuma, and K. Ishida, Study of Diffusion Mobility of Al-Zn Solid Solution, J. Phase Equilib. Diffus., 2006, 27, p 333-342

5. L.J. Zhang, Y. Du, Q. Chen, I. Steinbach, and B.Y. Huang, Atomic Mobilities and Diffusivities in the fcc, L12 and B2 Phases of the Ni-Al System, Int. J. Mater. Res., 2010, 101, p 1461-1475

6. G. Ghosh, Thermodynamic and Kinetic Modeling of the Cr-Ti-V System, J. Phase Equilib., 2002, 23, p 310-328

7. Y. Liu, Y. Ge, D. Yu, T. Pan, and Z. Lijun, Assessment of the Diffusional Mobilities in High-Strength Ti-V Alloys, J. Alloy. Compd., 2009, 470, p 176-182

8. A.T. Dinsdale, SGTE Data for Pure Elements, CALPHAD, 1991, 15, p 317-425

\section{E. Campbell}

Materials Science and Engineering Division

NIST, Gaithersburg

MD 20899-8555, USA 